\title{
Smooth Infinite Economies
}

\author{
by \\ Graciela Chichilnisky, Columbia University \\ Yuquing Zhou
}

\section{May 1996}

Discussion Paper Series No. 9596-17

gacs: 24 


\title{
Smooth Infinite Economies \\ Graciela Chichilnisky and YuQING ZhOU \\ PROGRAM ON INFORMATION AND RESOURCES \\ COLUMBIA UNIVERSITY \\ NEW YORK, NY 10027
}

May 23, 1996

\begin{abstract}
Equilibrium conditions in smooth infinite economies with separable utilities are described by Fredholm maps, which are Frechet differentiable. Therefore, Smale's extension of Sard's theorem can be used to studying infinite economies. We study structural stability and local uniqueness of equilibrium in smooth infinite economies and relate the theory of markets to modern Fredholm theory.
\end{abstract}




\section{INTRODUCTION}

Many problems in economics involve infinite dimensions. Typical examples are dynamic choices, such as optimal portfolios in finance and optimal paths in growth models (see, for example, Duffie and Huang [16], Chichilnisky and Kalman [10]). In the past two decades there has been an increasing interest in the study of infinite economies. However, much of the literature so far has mainly dealt with existence of equilibrium; determinacy of equilibrium is largely unexplored. Chichilnisky and Kalman [10] has dealt with a special case in the context of resource allocation problem. Kehoe et al [20] have followed an approach that takes excess demand functions as primitives. This paper proposes to attack the problem by taking preferences and endowments as primitives. We set up a framework for analysis and relate determinacy theory to modern Fredholm theory. This is useful because differential topology can be extended to infinite dimensional manifolds with Fredholm maps. Under these conditions a locally stable equilibrium corresponds to a point in the domain of some Fredholm map, at which the Fredholm index is equal to zero. The central issues in this approach are the following:

1. To identify a "right" framework, which is both mathematically tractable and economically meaningful. In other words, to choose a "right" topological space, where Fredholm theory can be applied and which is natural from economic point of view. This two requirements are usually not satisfied simultaneously. For example, the positive cone of Hilbert space $L^{2}$ is natural in financial markets but not good for Fredholm theory because it has an empty interior ${ }^{1}$.

2. To characterize conditions for the parameters of dynamic economic systems such that the underlying economies are "well behaved". To apply Fredholm theory, the excess demand function of the economy must be a Fredholm map between two

\footnotetext{
${ }^{1}$ See Chichilnisky [6], [7], [8] for the introduction of Hilbert space and Sobolev space (such as $L^{2}$ ) in economic models. In Fredholm theory, the domain and range of Fredholm maps must have the structure of Banach manifolds. Since $L_{+}^{2}$ has empty interior, it is not a Banach manifold, therefore, $L^{2}$ is not an ideal space for Fredholm theory because prices are always in $L_{+}^{2}$.
} 
infinite dimensional manifolds. This property can not be "assumed": it must be derived from the parameters of the economy. Even continuity is not guaranteed. Excess demand functions in infinite economies are typically not well-defined and are generally not smooth even when they are well-defined. Indeed, following Chichilnisky [6], and subsequently Araujo [2], excess demand functions could be smooth only if the commodity space is a Hilbert space, whose natural positive cone usually has empty interior.

In order to use differential techniques, we assume that the commodity space $L$ is a separable topological vector space for which the interior int $L^{+}$of the positive cone $L^{+}$is non-empty. Typically examples of such spaces are $C([0, T])$ with the supremum norm $\|x\|=\sup _{t \in[0, T]}|x(t)|$ and the positive cone $C^{+}([0, T])=$ $\{x: x(t) \geq 0, \forall t \in[0, T]\}$. For simplicity, in this paper we restrict ourself to the commodity space $C\left(M, R^{n}\right)$, where $M$ is any compact manifold. For more general treatment of commodity spaces, see Chichilnisky and Zhou [11].

The literature typically takes as the price space the natural positive cone $L^{*+}$ of the dual space $L^{*}$ of $L$. When the positive cone of the underlying commodity space has a non-empty interior, the corresponding price space is extremely large, which is the reason that excess demand functions cannot be well-defined. However, not all elements of the price space are equally interesting. With separable utility functions, only a small subset of the price space can support equilibria. Therefore, there is no loss of information from discarding those elements that do not support equilibria. This is the key step in our departure from the traditional way of thinking about this problem, which makes it possible to bring differential techniques to infinite spaces.

We show that, for separable utility functions on infinite dimensional Banach spaces, equilibrium conditions are described by Fredholm maps, which are Frechet differentiable. Therefore, Smale's extension of Sard's theorem can be used to obtain the determinacy theory of equilibrium ${ }^{2}$.

\footnotetext{
${ }^{2}$ In addition to the papers we have mentioned, there are other papers dealing with determinacy theory of equilibrium in infinite dimension, using different approaches. See, for example, Dana [13] and Shannon [22].
} 
The next section will set up a general framework we will work with. Sections 3 -5 report the main results in the paper. The last section makes some concluding remarks and lists our plans for further research.

\section{Preliminaries and mathematical notations}

In this section we group together some basic mathematical definitions, notations and facts that will be used later.

2.1. Fredholm index theory. The most important mathematical concept that we will use is Fredholm map. Here we only give a brief review. Readers may refer to Yosida [23] or Conway [12] for details.

Given two Banach spaces $X$ and $Y$, the vector space of all bounded linear maps from $X$ to $Y$ will be denoted by $L(X, Y)$, with the norm $\|$. $\|$ defined by

$$
\|T\|=\sup \{\|T x\|:\|x\| \leq 1\}
$$

$L(X, Y)$ is a Banach space. Let $M$ be a closed subspace of $X$. Define

$$
\operatorname{Codim} M=\operatorname{Dim}(X / M)
$$

Let $T \in L(X, Y)$, and

$$
\begin{aligned}
\operatorname{Ker} T & =\{x \in X, T x=0\} \\
\operatorname{Ran} T & =\{y \in Y, T x=y, x \in X\}
\end{aligned}
$$

The map $T$ is said to be a Fredholm operator if, and only if, RanT is closed and

$$
\operatorname{Dim}(\operatorname{Ker} T)<\infty, \operatorname{Codim}(\operatorname{Ran} T)<\infty
$$

The index of $T$ is defined by

$$
\operatorname{IndT}=\operatorname{Dim}(\operatorname{Ker} T)-\operatorname{Codim}(\operatorname{Ran} T)
$$

We denote by $D(X, Y) \in L(X, Y)$ all Fredholm operators. A linear map $T \in$ $L(X, Y)$ is compact if $T(x,\|x\| \leq 1)$ has compact closure in $Y$. The set of compact operators from $X$ into $Y$ is denoted by $L_{0}(X, Y) . T \in L(X, Y)$ has finite rank 
if $\operatorname{RanT}$ is finite dimensional. Clearly any linear map that has finite rank is compact. An interesting fact about Fredholm operators is that their index is invariant under compact perturbations.

Theorem 1. Let $A \in D(X, Y)$, and suppose that $K \in L_{0}(X, Y)$. Then $A+K \in$ $D(X, Y)$ and $\operatorname{Ind}(A+K)=\operatorname{Ind} A$

Theorem 2. Let $X, Y, Z$ be Banach spaces and suppose that $A \in D(X, Y)$ and $B \in D(Y, Z)$, then $A B \in D(X, Z)$ and

$$
\operatorname{Ind}(A B)=\operatorname{Ind} A+\operatorname{Ind} B
$$

Theorem 3. If $A$ is invertible and $K \in L_{0}(X, Y)$, then Ind $(A+K)=0$.

2.2. Basic nonlinear analysis. We assume the readers are familiar with basic calculus on Banach space. A basic reference is Zeidler [24], or any standard functional analysis textbooks.

First we note that there are similar implicit mapping and inverse mapping theorems on Banach spaces, see Abraham and Robbin [1]

Theorem 4 [Implicit mapping theorem]. Let $X, Y, Z$ be Banach spaces, $U$ is open in $X, V$ is open in $Y, f: U \times V \rightarrow Z$ be a $C^{m}$-map $(m>0)$. Let $f_{2}^{\prime}\left(x_{0}, y_{0}\right): Y \rightarrow Z$ be a homeomorphism for $(x, y) \in U \times V$, where $f_{2}^{\prime}\left(x_{0}, y_{0}\right)$ represents the partial derivative of $f$ with respect to the second variable $y$. Then there is an open neighborhood $U_{0}$ of $x_{0}$ and $C^{m}$-map $g: U_{0} \rightarrow V$ such that $g\left(x_{0}\right)=y_{0}$ and $f(x, g(x))=f\left(x_{0}, y_{0}\right)$ for all $x \in U_{0} . g$ is unique

Theorem 5 [Inverse mapping theorem]. Let $X, Y, Z$ be Banach spaces, $U$ is open in $X, f: U \rightarrow Z$ be a $C^{m}$-map $(m>0)$. If $f^{\prime}\left(x_{0}\right)$ is a homeomorphism, then there is a neighborhood $U_{0}$ of $f\left(x_{0}\right)$ in $U$ and a unique $C^{m}$-map $g: U_{0} \rightarrow Y$ such that $g$ is a homeomorphism of $U_{0}$ onto an open neighborhood of $x_{0}$ in $Y$. 
Let $X$ and $Y$ be Banach manifolds and $f: X \rightarrow Y$ be a $C^{1}$-map. The Frechet derivative of $f$ is denoted by $f^{\prime}$, and the "differential" of $f$ is denoted by $D f .{ }^{3}$ We shall say that $f$ is a Fredholm map if for all $x \in X$, the linear map $f^{\prime}(x): T_{x} X \rightarrow T_{f(x)} Y$ is a Fredholm operator. If $X$ is connected, the index of $f^{\prime}(x)$ will not depend on the particular choice of the point $x$ in $X$ and is referred to the index of $f$. The following Sard-Smale's theorem will be the main tool to explore the generic property of local uniqueness of competitive equilibrium.

Theorem 6 [Sard-Smale's theorem]. Let $f: X \rightarrow Y$ be a $C^{m}(m>0)$ Fredholm map between separable Banach manifolds $X$ and $Y$. Assume $Y$ is complete, $X$ connected, and $m>$ index $(f)$. Then the set of regular values for $f$ contains a dense $G_{\delta}$ subset of $Y . A G_{\delta}$ subset is defined as the intersection of a countable family of open subsets.

\section{The MARKeT}

The model represents a pure exchange economy with infinitely many commodities and a finite number of consumers. It is derived from economic considerations and can be summarized as follows:

The economy is denoted

$$
\Im=\left(W_{i}, \varpi_{i}, X_{i}\right)_{1 \leq i \leq I}
$$

There are $I$ agents, index by $i . X_{i}$ is the consumption set of agent $i$. For simplicity, in this paper we may assume that $X_{i}=C^{++}\left(M, R^{n}\right) . W_{i}(x)$ is the utility function of agent $i . \varpi_{i}$ is the initial endowment of agent $i$. Society's endowment $\varpi=\sum_{i}^{I} \varpi_{i} \epsilon$ $C^{++}\left(M, R^{n}\right)$. We assume that the utility functions are separable, i.e., they can be written as the following form

$$
W_{i}(x)=\int_{M} u^{i}(x(t), t) d t .
$$

\footnotetext{
${ }^{3}$ For a definition of the Frechet derivative and the differential of a map on Banach manifolds, see Abraham and Robbin [1], or Zeidler [24].
} 
where the integral is with respect to some metric on the compact manifold $M^{4}$.

Example 1. In growth models, the utility function $W_{i}(x)$ is simply a continuoustime version of a discounted sum of time-dependent utilities. In finance, when the underlying parameters follow a diffusion process, $W_{i}(x)$ is just the expectation of state-dependent utilities, and $M$ is the state space.

Since we are going to use differential techniques, we need to find conditions that guarantee that $W_{i}(x)$ is twice Frechet differentiable. Let there be given $C^{2}$ functions

$$
u^{i}(x, t): R_{++}^{n} \times M \rightarrow R
$$

Given $t$, let

$$
V_{t}^{i}(x)=\left\{y \in R_{++}^{n} \mid u^{i}(y, t) \geq u^{i}(x, t)\right\}
$$

Assumption 1. For any fixed $t \in M$, the function $u^{i}(x,):. R_{++}^{n} \rightarrow R$ is strictly monotonic and concave, and $V_{t}^{i}(x)$ is closed.

Proposition 1. Under the assumption 1, the function

$$
W_{i}(x)=\int_{M} u^{i}(x(t), t) d t
$$

is strictly monotonic, concave over $X_{i}$, and is twice Frechet differentiable.

Proof. The proof has several steps:

Step 1: We observe that

$$
W_{i}(x)=\int_{M} u^{i}(x(t), t) d t
$$

is well-defined. It is a simple consequence of Assumption 1.

Step 2: We show that $W_{i}(x)$ is a $C^{1}$ functional. Take any $x(t) \in C^{++}\left(M, R^{n}\right)$ and write the Gateaux derivative with respect to $v(t) \in C^{++}\left(M, R^{n}\right)$.

$$
\frac{d W_{i}(x+a v)}{d a}=\frac{\partial}{\partial a} \int_{M} u^{i}(x(t)+a v(t), t) d t
$$

\footnotetext{
${ }^{4}$ The assumption of compactness is only for the simplicity of exposition, it is by no means necessary. For a general treatment, see Chichilnisky and Zhou [11].
} 
We can use the Lebesgue theorem on the differentiation of integrals with respect to a parameter

$$
\begin{aligned}
& \frac{\partial}{\partial a} \int_{M} u^{i}(x(t)+a v(t), t) d t \\
= & \int_{M} \frac{\partial}{\partial a} u^{i}(x(t)+a v(t), t) d t
\end{aligned}
$$

Therefore

$$
\begin{aligned}
\left.\frac{d W_{i}(x+a v)}{d a}\right|_{a=0} & =\int_{M} u_{x}^{i}(x(t), t) v(t) d t \\
& =\left\langle u_{x}^{i}(x(t), t), v(t)\right\rangle
\end{aligned}
$$

So under the inner product ${ }^{5}\langle.,$.$\rangle on C\left(M, R^{n}\right)$, the Frechet derivative of $W_{i}(x)$, denoted by $W_{i}^{\prime}(\cdot)$, can be uniquely expressed as the following form:

$$
W_{i}^{\prime}(x(t))=u_{x}^{i}(x(t), t)
$$

Step 3: To show that $W_{i}^{\prime}(x(t))$ is $C^{1}$ Frechet differentiable, we recall the definition of Frechet derivative.

Definition 1. Given a map $\pi: X \rightarrow Y$, a point $x \in X$ and a map $A \in L(X, Y)$, we say that $A$ is the Frechet derivative of $\pi$ at $x$ if

$$
\lim _{y \rightarrow 0} \frac{1}{\|y\|}\|\pi(x+y)-\pi(x)-A y\|=0
$$

where $X$ and $Y$ are Banach spaces.

Now we return to the proof of step 3 .

First, we show that

$$
W_{i}^{\prime}(x(t)): x(t) \rightarrow u_{x}^{i}(x(t), t)
$$

is continuous.

\footnotetext{
${ }^{5}$ We observe that it is not complete.
} 
Let $x_{n}(t) \rightarrow x(t)$ in $C\left(M, R^{n}\right)$, we need to show that

$$
u_{x}^{i}\left(x_{n}(t), t\right) \rightarrow u_{x}^{i}(x(t), t)
$$

is in $C\left(M, R^{n}\right)$. Since $|x(t)|<b$ for some constant $b, u_{x}^{i}(x(t), t)$ is also bounded by the compactness of $M$. Therefore,

$$
\begin{aligned}
& \left\|u^{i}\left(x_{n}(t), t\right)-u^{i}(x(t), t)\right\| \\
\leq & \bar{b}\left\|x_{n}(t)-x(t)\right\| \rightarrow 0
\end{aligned}
$$

Now we show that $W_{i}^{\prime}(x(t))$ is Frechet differentiable and that

$$
W_{i}^{\prime \prime}(x(t))=u_{x x}^{i}(x(t), t)
$$

Following the definition, we need to show that

$$
\lim _{y \rightarrow 0} \frac{1}{\|y\|}\left\|u_{x}^{i}(x(t)+y(t), t)-u_{x}^{i}(x(t), t)-u_{x x}^{i}(x(t), t) \circ y(t)\right\|=0
$$

We note that $u_{x x}^{i}(x(t), t)$ is a matrix-valued function of $t$ and that

$$
u_{x}^{i}(x(t)+y(t), t)-u_{x}^{i}(x(t), t)=u_{x x}^{i}(x(t)+\theta(t), t) \circ y(t)
$$

where $0<\theta(t)<y(t)$. So the above formula can be written as

$$
\begin{aligned}
& \lim _{y \rightarrow 0} \frac{1}{\|y\|}\left\|\left[u_{x x}^{i}(x(t)+\theta(t), t)-u_{x x}^{i}(x(t), t)\right] \circ y(t)\right\| \\
\leq & \lim _{y \rightarrow 0}\left\|\left[u_{x x}^{i}(x(t)+\theta(t), t)-u_{x x}^{i}(x(t), t)\right]\right\| \\
= & 0
\end{aligned}
$$

since $\theta(t) \rightarrow 0$ when $y(t) \rightarrow 0$ in $\|$.$\| -norm.$

\section{EXCESS DEMAND FUNCTIONS}

An excess demand function is a map from the price space to the commodity space describing the difference between what is available and what is demanded by all traders at each price. The demand function describes what traders desire and can afford to buy at those prices. In finite $L$-dimensional spaces, excess demand functions are formally defined as follows: 
Definition 2. A function $f_{i}: R_{++}^{L} \rightarrow R^{L}$ is called the excess demand function of agent $i$ if it is defined by letting $x_{i}=f_{i}(p)+\varpi_{i}$ be the unique maximizer ${ }^{6}$ of $W_{i}\left(x_{i}\right)$ subject to the budget constraint $p x_{i} \leq p \varpi_{i}$.

In the case of infinite dimensional spaces, the existence of excess demand functions can be problematic. The literature typically takes as the price space the natural positive cone of the dual space of the underlying commodity space. When the positive cone of the underlying commodity space has non-empty interior, the correspondent price space is extremely large. In this case excess demand functions in infinite economies typically are not well defined.

However, not all elements of the price space are equally interesting. With separable utility functions, only a small subset of the price space can support equilibria. There is no loss of information from discarding away those elements that do not support equilibria. This is the key step in our departure from the traditional way of thinking about this problem, which makes it possible to bring the differential techniques to infinite spaces.

In this section, we will develop this approach. Unlike the current literature, we will restrict our attention to a subset of the price space to define an excess demand function without loss of information. To simplify the exposition, we make the following assumptions:

Assumption 2: When $n=1$,

$$
\lim _{x \rightarrow 0^{+}} u_{x}^{i}(x, t)=+\infty
$$

for each fixed $t$.

Remark 1. Assumption 2 is widely used in financial literature to obtain interior equilibria. It assumes that instantaneous utility functions have infinite marginal utility for consumption at zero.

\footnotetext{
${ }^{6}$ The uniqueness is not required in the usual definition of excess demand function. It can be guaranteed by the assumption of strict concavity of utility functions.
} 
When $n>1$, we would like to make a similar assumption for utility functions. For each fixed $t$, consider the corresponding indirect utility functions. Denote by $V(p, w, t)=u(x(p, w), t)$ the indirect utility functions, where

$$
x(p, w)=\arg \max _{p x \leq w} u(x, t), x \in R^{n}, p \in R_{++}^{n}
$$

For each fixed $p$ and $t$, we know $\frac{\partial V}{\partial w}>0$.

Assumption 3: When $n>1$,

$$
\lim _{w \rightarrow 0^{+}} \frac{\partial V}{\partial w}=+\infty
$$

for each fixed $t$ and $p$.

Proposition 2. Let

Under assumptions 1-3,

$$
\operatorname{Grad}_{i}(x(t))=\frac{u_{x}^{i}(x(t), t)}{\left\|u_{x}^{i}(x(t), t)\right\|}
$$

$$
\operatorname{Grad} W_{i}(x(t)): C^{++}\left(M, R^{n}\right) \rightarrow S_{++}
$$

is $C^{1}$ map and onto, where $S_{++}=\left\{y \in C^{++}\left(M, R^{n}\right) \mid\|y\|=1\right\}$

Proof. Let $p(t) \in S_{++}$, and choose some $\lambda>0$ such that

$$
\min _{t \in M} \lambda p(t)
$$

is sufficiently large. Let

$$
u_{x}^{i}(x(t), t)=\lambda p(t)
$$

By our assumptions, there is a well-defined $x(t)$ such that the above formula holds. Now we need to show that such $x(t)$ is contained in $C^{++}\left(M, R^{n}\right)$. To see this, we consider the inverse of $u_{x}^{i}(x(t), t)$ with respect to $t$,

$$
x(t)=\left(u_{x}^{i}\right)^{-1}(\lambda p(t), t)
$$

By the implicit mapping theorem, we know that $\left(u_{x}^{i}\right)^{-1}(c, t)$ is continuous with respect to $c$ and $t$. The same procedure as in the proof of Proposition 1 completes the proof. 
Definition 3. A price $P: C\left(M, R^{n}\right) \rightarrow R$ is real valued linear function on $C\left(M, R^{n}\right)$ which gives nonnegative value to any element in $C^{++}\left(M, R^{n}\right)$. A feasible allocation $\vec{x}(t)=\left(x_{1}(t), \ldots, x_{I}(t)\right)$ is an allocation such that $\sum_{i=1}^{I} x_{i} \leq \varpi$. A feasible allocation is an equilibrium when there is a nonzero price $P$ with $P\left(\varpi_{i}\right)=P\left(x_{i}\right)$ and for any $y \in C^{++}\left(M, R^{n}\right)$,

$$
W_{i}(y)>W_{i}\left(x_{i}\right) \rightarrow P(y)>P\left(x_{i}\right)
$$

Existence of equilibrium can be derived from Bewley $[5]^{7}$. It is shown in Bewley [5] that, to obtain equilibrium prices in $L_{1}(M, \Sigma, \mu)$ (a subset of the dual space of $\left.L_{\infty}(M, \Sigma, \mu)\right),{ }^{8}$ utility functions must be Mackey continuous ${ }^{9}$. He showed that this condition, in addition to the basic assumptions, is indeed sufficient.

Theorem 7 [Bewley]. Assume, in addition to the assumptions 1-3, that

(i) each utility function is Mackey continuous;

(ii) $\varpi \in i n t L_{\infty}^{+}(M, \Sigma, \mu)$.

Then the economy has a quasi-equilibrium, and every quasi-equilibrium price belongs to $L_{1}(M, \Sigma, \mu)$.

It is shown in Bewley [5] that separable utility functions considered in this paper are indeed Mackey continuous. Furthermore, from the discussions in previous sections and under assumptions 1-3, it is easy to show that every quasi-equilibrium is an equilibrium and every equilibrium price belongs to $\operatorname{int} L_{\infty}^{+}(M, \Sigma, \mu)$. In the following we further refine Bewley's result. We note that our consumption space $C^{++}\left(M, R^{n}\right)$ can be embedded into $L_{\infty}^{+}(M, \Sigma, \mu)$. In our case, the state space $M$ is a compact manifold, the $\sigma$-algebra $\Sigma$ is generated by the open sets of $M$, and the probability measure $\mu$ is the given metric on $M$ (of course, we can normalize $\mu$ such that $\mu(M)=$ 1).

\footnotetext{
${ }^{7}$ For a more general discussion of existence of equilibrium in Hilbert space and Sobolev space, see Chichilnisky and Heal [8].

${ }^{8} L_{\infty}(M, \Sigma, \mu)$ consists of all essentially bounded functions from $M$ to $R^{n}$.

${ }^{9}$ The Mackey topology is the strongest topology on $L_{\infty}$, for which $L_{1}$ is the dual of $L_{\infty}$.
} 
Theorem 8 [Refinement of Bewley]. If $\varpi_{i} \in C^{++}\left(M, R^{n}\right), \forall i$, then, under assumption 1-3, every equilibrium price $p \in C^{++}\left(M, R^{n}\right)$ and every equilibrium consumption $c_{i}$ of agent $i$ belongs to $C^{++}\left(M, R^{n}\right)$.

Proof. Let $u_{i}\left(c_{i}, t\right)=\lambda_{i} p, \forall i$. It is easy to see that $\lambda_{i} \neq 0$. A competitive equilibrium is Pareto efficient by the first welfare theorem, therefore it maximizes a weighted sum of individual utilities. For separable preferences, the weight is just the inverse of the $\lambda_{i}^{\prime}$ s at the optimal consumption. ${ }^{10}$ Let

$$
\bar{u}(y, t)=\max _{x_{i} \in L_{\infty}^{+}} \sum_{i=1}^{I} \frac{1}{\lambda_{i}} u_{i}\left(x_{i}, t\right)
$$

subject to

$$
\sum_{i=1}^{I} x_{i} \leq y .
$$

It is easily verified that $\bar{u}(y, t)$ satisfies all assumptions about utility functions in previous sections. Let

$$
\bar{U}(y)=\int_{M} \bar{u}(y(t), t) d t
$$

We note that

$$
\bar{u}^{\prime}(\varpi, t)=\lambda p
$$

for some $\lambda>0$. Therefore, $\varpi \in C^{++}\left(M, R^{n}\right)$ implies that $p \in C^{++}\left(M, R^{n}\right)$.

The next result, combined with proposition 2, tells us that we can establish similarly individual demand theory in infinite economies for the model specified in this paper.

Proposition 3. For all $p(t) \in S_{++}$, and $w \in R_{++}$, under assumptions 1-3, there is a unique demand vector $F_{i}(p(t), w) \in C^{++}\left(M, R^{n}\right)$ such that

$$
F_{i}(p(t), w)=\underset{\langle p(t), y\rangle=w}{\arg } \max W_{i}(y)
$$

\footnotetext{
${ }^{10}$ See Chi-Fu Huang [19].
} 
If and only if

$$
\begin{array}{r}
u_{x}^{i}\left(F_{i}(p(t), w), t\right)=\lambda p(t) \\
\left\langle p(t), F_{i}(p(t), w)\right\rangle=w
\end{array}
$$

for some $\lambda>0$, and furthermore,

$$
F_{i}(p(t), t): S_{++} \times R_{++} \rightarrow C^{++}\left(M, R^{n}\right)
$$

is a diffeomorphism.

Proof. Step 1: Necessity.

It is an immediate consequence of Proposition 2.

Step 2: Sufficiency.

Denote the hyperplane by

$$
A=\left\{y \in C^{++}\left(M, R^{n}\right) \mid\langle p(t), y\rangle=w\right\}
$$

Obviously, $W_{i}(y)$ is a strictly concave function on $A$. Also,

$$
\begin{aligned}
\left.\frac{d W_{i}\left(F_{i}+a\left(y-F_{i}\right)\right)}{d a}\right|_{A} & =\int_{M} u_{x}^{i}\left(F_{i}, t\right)\left(y-F_{i}\right) d t \\
& =\lambda \int_{M} p(t)\left(y-F_{i}\right) d t \\
& =0
\end{aligned}
$$

Therefore,

$$
F_{i}(p, w)=\underset{y \in A}{\arg \max } W_{i}(y)
$$

It is unique globally on $A$ by the strict concavity of $W_{i}(y)$.

Step 3: Denote $G_{i}: C^{++}\left(M, R^{n}\right) \rightarrow S_{++} \times R_{++}$by

$$
G_{i}(x(t))=\left[\operatorname{Grad} W_{i}(x(t)),\left\langle\operatorname{Grad} W_{i}(x(t)), x(t)\right\rangle\right]
$$

It is easy to check that $G_{i}$ is differentiable and onto. From step 2 , we know that the composite map

$$
F_{i} \circ G_{i}: C^{++}\left(M, R^{n}\right) \rightarrow C^{++}\left(M, R^{n}\right)
$$


is identity map, and

$$
G_{i} \circ F_{i}: S_{++} \times R_{++} \rightarrow S_{++} \times R_{++}
$$

is also identity map. Therefore, $F_{i}^{-1}=G_{i}$.

Proposition 4. Under assumptions 1-3, the demand function is a Fredholm map and the index of $F_{i}^{\prime}(p, w)$ at $(p, w)$ is 0 . Furthermore, the index of $F_{i}^{\prime}(p, w)$ can be written as the sum of a inverse operator and a finite rank operator.

Proof. Ind $F_{i}^{\prime}=0$ is a direct result of Proposition 3. Now let's calculate $F_{i}^{\prime}$ by means of the formula in Proposition 3. Denote by $D F_{i}$ the differential of $F_{i}$. Differentiate the two sides of the following two equations

$$
\begin{aligned}
u_{x}^{i}\left(F_{i}, t\right) & =\lambda p(t) \\
\left\langle p(t), F_{i}\right\rangle & =w
\end{aligned}
$$

we get

$$
\begin{aligned}
u_{x x}^{i}\left(F_{i}, t\right) D F_{i} & =\lambda D p(t)+D \lambda p(t) \\
\left\langle D p(t), F_{i}\right\rangle+\left\langle p(t), D F_{i}\right\rangle & =D w
\end{aligned}
$$

simplifying the above formula, we get

$$
D F_{i}=\lambda\left(u_{x x}^{i}\right)^{-1} D p(t)+D \lambda\left(u_{x x}^{i}\right)^{-1} p(t)
$$

Where $\left(u_{x x}^{i}\right)^{-1}$ is the inverse of matrix $u_{x x}^{i}\left(F_{i}, t\right)$ for each $t$. Put (2) into (1), we obtain

$$
\begin{aligned}
D \lambda & <p(t),\left(u_{x x}^{i}\right)^{-1} p(t)> \\
& =-\lambda<p(t),\left(u_{x x}^{i}\right)^{-1} D p(t)>-\left\langle D p(t), F_{i}\right\rangle+D w
\end{aligned}
$$

Therefore,

$$
D \lambda=-\left[\frac{\lambda\left\langle p(t),\left(u_{x x}^{i}\right)^{-1} D p(t)\right\rangle+\left\langle D p(t), F_{i}\right\rangle-D w}{\left\langle p(t),\left(u_{x x}^{i}\right)^{-1} p(t)\right\rangle}\right]
$$


Therefore, substituting $D \lambda$ in (2) by (3), we obtain

$$
\begin{aligned}
D F_{i}(p(t), w)= & -\frac{\lambda\left\langle p(t),\left(u_{x x}^{i}\right)^{-1} D p(t)\right\rangle+\left\langle D p(t), F_{i}\right\rangle}{\left\langle p(t),\left(u_{x x}^{i}\right)^{-1} p(t)\right\rangle}\left(u_{x x}^{i}\right)^{-1} p(t) \\
& +\frac{\left(u_{x x}^{i}\right)^{-1} p(t)}{\left\langle p(t),\left(u_{x x}^{i}\right)^{-1} p(t)\right\rangle} D w+\lambda\left(u_{x x}^{i}\right)^{-1} D p(t)
\end{aligned}
$$

From (4) the operator defined by the first term has a finite rank, and the sum of the last two terms defines an invertible operator, therefore, $F_{i}$ is, by definition, a Fredholm operator.

\section{Stability of Equilibrium}

Now we have all the required mathematical tools in our hand to deal with the main issue in this paper. Since for each agent $i$, there is a well-defined individual demand function $F_{i}(p(t), w)$, we can define our economy by $\bar{\Im}=\left(F_{i}, X_{i}, \varpi_{i}\right)_{1 \leq i \leq I}$. For the rest of the section, we will denote by $H=C^{++}\left(M, R^{n}\right)$. We assume that the demand functions are fixed, so the economy is actually defined by

$$
\varpi=\left(\varpi_{1}, \ldots, \varpi_{I}\right) \in \underbrace{H \times \cdots H}_{I}=H^{I}
$$

Given $\varpi \in H$, if an element $p(t) \in H$ satisfying

$$
\sum_{i=1}^{I} F_{i}\left(p(t),\left\langle p(t), \varpi_{i}\right\rangle\right)=\sum_{i=1}^{I} \varpi_{i}
$$

then $p(t)$ is an equilibrium price of the economy. We denote by $E(\varpi)$ the set of $p(t)$ satisfying the above equality.

Theorem 9 [The main theorem]. There is a dense $G_{\sigma}$ subset $V$ of $H^{I}$, the space of endowments, such that $E(\varpi)$ is discrete for any $\varpi \in V$, and for each such discrete point $\varpi$, locally the equilibrium in $E(\varpi)$ depends continuously on $\varpi$.

Proof. An outline of the proof is as follows. We show that the function $F$ as defined below is a Fredholm map, therefore, we can use the Sard-Smale theorem and 
follow directly the proof for the finite dimensional case in Debreu [15]. Let

$$
\Delta=S_{++} \times R_{++} \times H^{I-1}
$$

We define the function $F$ from $\Delta$ to $H^{I}$ by associating with an element $e=\left(p(t), w, \varpi_{2}, \ldots \varpi_{I}\right)$ of $\Delta$ the value $F(e)=\left(\varpi_{1}, \ldots, \varpi_{I}\right)$, where

$$
\varpi_{1}=F_{1}(p(t), w)+\sum_{i=2}^{I} F_{i}\left(p(t),\left\langle p(t), \varpi_{i}\right\rangle\right)-\sum_{i=2}^{I} \varpi_{i}
$$

It is a simple exercise to find that for every $e \in \triangle$, one has $\left\langle p(t), \varpi_{1}\right\rangle=w$. We also note that, given $\varpi \in H^{I}$, the equilibrium price $p(t)$ belongs to $E(\varpi)$ if and only if

$$
F\left(p(t),\left\langle p(t), \varpi_{1}\right\rangle, \varpi_{2}(t), \varpi_{3}(t), \ldots, \varpi_{I}(t)\right)=\varpi(t)
$$

and that the points of $E(\varpi)$ are in one-to-one correspondence with the points of $F^{-1}(\varpi)$. From the last section, we know that $F$ is differentiable. In order to use Smale's theorem, we need to prove that the index of $F^{\prime}$ is 0 .

To see this, let $\varpi_{-1}=\left(\varpi_{2}, \ldots, \varpi_{I}\right)$. Therefore,

$$
F:\left(p(t), w, \varpi_{-1}\right) \rightarrow\left(\varpi_{1}, \varpi_{-1}\right)
$$

So we have

$$
F^{\prime}:\left(T_{p(t)}, R, \varpi_{-1}\right) \rightarrow\left(\varpi_{1}, \varpi_{-1}\right)
$$

Let

$$
\frac{\partial F}{\partial(p(t), w)}:\left(T_{p(t)}, R\right) \rightarrow\left(\varpi_{1}, \varpi_{-1}\right)
$$

be the partial derivative with respect to $(p(t), w)$, while $\varpi_{-1}$ remains fixed. In the same way, we define the partial derivative $\frac{\partial F}{\partial_{\varpi_{-1}}}$ with respect to $\varpi_{-1}$. We directly calculate the partial differential of $D_{(p(t), w)} F$ and $D_{\varpi_{-1}} F$ as follows

$$
D_{(p(t), w)} F=D F_{1}+\sum_{i=2}^{I}\left[\frac{\partial F_{i}}{\partial p} D p(t)+\frac{\partial F_{i}}{\partial w}\left\langle D p(t), \varpi_{i}\right\rangle\right]
$$


From the equality 4 in the last section, we have

$$
\begin{aligned}
D_{(p(t), w)} F= & {\left[\sum_{i=1}^{I} \lambda_{i}\left(u_{x x}^{i}\right)^{-1}\right] D p(t)+} \\
& {\left[\sum_{i=1}^{I} \frac{-\lambda_{i}\left\langle p(t),\left(u_{x x}^{i}\right)^{-1} D p(t)\right\rangle-\left\langle D p(t), F_{i}\right\rangle+\left\langle D p(t), \varpi_{i}\right\rangle}{\left\langle p(t),\left(u_{x x}^{i}\right)^{-1} p(t)\right\rangle}\left(u_{x x}^{i}\right)^{-1}\right] p(t)+} \\
& \frac{D w-\left\langle D p(t), \varpi_{1}\right\rangle}{\left\langle p(t),\left(u_{x x}^{1}\right)^{-1} p(t)\right\rangle}\left(u_{x x}^{1}\right)^{-1} p(t)
\end{aligned}
$$

Since for each fixed $t,\left(u_{x x}^{i}\right)^{-1}$ is a negative definite symmetric matrix for each fixed $t$, hence

$$
Z=\sum_{i=1}^{I} \lambda_{i}\left(u_{x x}^{i}\right)^{-1}: H \rightarrow H
$$

is an invertible linear operator. As for $D_{\varpi_{-1}} F$,

$$
D_{\varpi_{-1}} F=\sum_{i=2}^{I}\left[\frac{\partial F_{i}}{\partial w}\left\langle p(t), D \varpi_{i}\right\rangle\right]-D \varpi_{-1}
$$

Therefore, we have

$$
\begin{aligned}
D F\left(p(t), w, \omega_{-1}\right)= & \left\{Z D p(t)+\frac{\left(u_{x x}^{1}\right)^{-1} p(t)}{\left\langle p(t),\left(u_{x x}^{1}\right)^{-1} p(t)\right\rangle} D w-D \varpi_{-1}\right\}+ \\
& {\left[\sum_{i=1}^{I} \frac{-\lambda_{i}\left\langle p(t),\left(u_{x x}^{i}\right)^{-1} D p(t)\right\rangle-\left\langle D p(t), F_{i}\right\rangle+\left\langle D p(t), \varpi_{i}\right\rangle}{\left\langle p(t),\left(u_{x x}^{i}\right)^{-1} p(t)\right\rangle}\left(u_{x x}^{i}\right)^{-1}\right] p(t) } \\
& \frac{-\left\langle D p(t), \varpi_{1}\right\rangle}{\left\langle p(t),\left(u_{x x}^{1}\right)^{-1} p(t)\right\rangle}\left(u_{x x}^{1}\right)^{-1} p(t)+\sum_{i=2}^{I}\left[\frac{\partial F_{i}}{\partial w}\left\langle p(t), D \varpi_{i}\right\rangle\right]
\end{aligned}
$$

The term in the bracket \{\} defines an invertible linear operator. The rest defines a finite rank linear operator. So the index of $F^{\prime}$ is 0 .

The rest of the proof is a simple application of Sard-Smale's theorem (see theorem 6 in section 2). See also Sard [21], Hirsch [18].

\section{FurThER RESEARCH}

Our approach yields useful results. They are summarized as follows: 
1. We have assumed separability, which is widely used in the literature. With this assumption, most price vectors can not support equilibria for the underlying economies. Therefore, it is safe to remove the set of prices that can not support equilibria, without loss of any information about the set of equilibria. A key result in this paper is that, on this price space, the excess demand function is a Fredholm map with index zero. This makes the application of Sard-Smale's theorem possible.

2. The index of Fredholm maps can be explicitly calculated (see Proposition 4 above). This makes the set of equilibria more tractable mathematically (see Theorem 9 above).

It seems possible to extend the above results to the following cases:

1. A continuum of agents. The set of traders $\{1, \ldots, I\}$ is replaced by a unit interval. If agents in the economies are not "too diverse" in a proper sense, our results should be applicable.

2. More general utility functions. The assumption of separability is by no means necessary. Many utility functions, such as those which exhibit "habit formation" (see Heal and Ryder [17]), or those which are called stochastic differential utility functions (see Duffie and Epstein [14]), have similar regular properties.

3. Other topological spaces. For example, the main theorem also applies to Sobolev spaces.

4. The topological structure of the set of equilibria. This describes the set of solutions to simultaneous nonlinear operators as the parameters of the economy vary. This set has been classified topologically in finite dimensional spaces (see Balasko [3], [4] for complete markets, and Chichilnisky and Heal [9] for incomplete markets) but not in infinite spaces. Using the techniques developed here it should be possible to obtain a complete characterization of the equilibrium manifold with infinite goods for both complete and incomplete markets.

\section{REFERENCES}

[1] R. Abraham and J. Robbin. Transversal Mappings and Flows. Benjamin, New York, 1967. 
[2] A. Araujo. The non-existence of smooth demand in general banach spaces. Journal of Mathematical Economics, 17:1-11, 1987.

[3] Y. Balasko. The graph of the walras correspondence. Econometrica, 43 (1975):907-912.

[4] Y. Balasko. Foundations of the Theory of General Equilibrium. Academic Press, Boston, MA, 1988.

[5] T. Bewley. Existence of equilibria in economies with infinitely many commodities. Journal of Economic Theory, 43:514-540, 1972.

[6] G. Chichilnisky. Manifolds of preferences and equilibria. $\mathrm{PhD}$ thesis, Department of Economics, University of California, Berkeley, 1976.

[7] G. Chichilnisky. Nonlinear functional analysis and optimal economic growth. Journal of Mathematical Analysis and Applications, Nov., 1977.

[8] G. Chichilnisky and G. M. Heal. Existence of a competitive equilibrium in sobolev spaces without bounds on short sales. Journal of Economic Theory, 59:364-384, 1993.

[9] G. Chichilnisky and G. M. Heal. The existence and structure of the pseudoequilibrium manifold in incomplete asset markets. Journal of Mathematical Economics, forthcoming, 1996.

[10] G. Chichilnisky and P. Kalman. An application of functional analysis of model of optimal allocation of resources with an infinite horizon. Journal of Optimization Theory and Applications, Jan., 1980.

[11] G. Chichilnisky and Y. Zhou. Nonlinear functional analysis and the theory of markets. preprint, Columbia University, 1996.

[12] J. B. Conway. A Course In Functional Analysis. Springer-Verlag, 1985. 
[13] R. A. Dana. Existence, uniqueness and determinacy of arrow-debreu equilibria in finance models. Journal of Mathematical Economics, 22:563-579, 1993.

[14] D.Duffie and L. G. Epstein. Stochastic differential utility. Econometrica, 60:353394, 1992.

[15] G. Debreu. Economies with a finite set of equilibria. Econometrica, 38 (1970):387-392.

[16] D. Duffie and C. F. Huang. Implementing arrow-debreu equilibria by continuous trading of few long-lived securities. Econometrica, 53:1337-1356, 1985.

[17] G. M. Heal and H. E. Ryder. Optimal growth with intertemporally dependent preferences. Review of Economic Studies, 40:1-31, 1973.

[18] M. Hirsch. Differential Topology. Springer-Verlag, New York, 1976.

[19] C. F. Huang. An intertemporal general equilibrium asset pricing model: the case ofdiffusion information. Econometrica, 55:117-142, 1987.

[20] T. J. Kehoe, D. K. Levine, A. Mas-Colell, and W. Zame. Determinacy of equilibrium in large square economies. Journal of Mathematical Economics, 18:231-262, 1989.

[21] A. Sard. The measure of the critical points of differentiable maps. Bulletin of the America Mathematical Society, 48:883-890, 1942.

[22] C. Shannon. Determinacy in infinite horizon exchange economies. Working paper, University of California at Berkeley, No. 94-233, December 1994.

[23] K. Yosida. Functional Analysis. Springer International, 1974.

[24] E. Zeidler. Nonlinear Functional Analysis and Its Applications, volume 1-5. New York, Springer-Verlag, 1985. 


\title{
1995-1996 Discussion Paper Series
}

\author{
Department of Economics \\ Columbia University \\ 1022 International Affairs Bldg. \\ 420 West 118th Street \\ New York, N.Y., 10027
}

The following papers are published in the 1995-96 Columbia University Discussion Paper series which runs from early November to October 31 of the following year (Academic Year).

Domestic orders for discussion papers are available for purchase at the cost of $\$ 8.00$ (U.S.) Per paper and $\$ 140.00$ (US) for the series.

Foreign orders cost $\$ 10.00$ (US) per paper and $\$ 185.00$ for the series.

To order discussion papers, please write to the Discussion Paper Coordinator at the above address along with a check for the appropriate amount, made payable to Department of Economics, Columbia University. Please be sure to include the series number of the requested paper when you place an order. 


\section{5-96 Discussion Paper Series}

9596-01 Protectionist Response to Import Competition in Declining Industries Reconsidered

9596-02 New Estimates on Climate Demand: Evidence from Location Choice

9596-03 Enforcement by Hearing

9596-04 Preferential Trading Areas and Multilateralism: Strangers, Friends or Foes?

9596-05 Simplification, Progression and a Level Playing Field

9596-06 The Burden of Proof in Civil Litigation

9596-07 Market Structure and the Timing of Technology Adoption

9596-08 The Emergence of the World Economy

9596-09 The Global Age: From a Skeptical South to a Fearful North

9596-10 A Conformity Test for Cointegration

9596-11 Identification and Kullback Information in the GLSEM

9596-12 Informational Leverage and the Endogenous Timing of Product Introductions

9596-13 Changes in Wage Inequality

9596-14 The Design of Monte Carlo Experiments for VAR Models

9596-15 A Toplogical Invariant for Competitive Markets

9596-16 Topology and Invertible Maps

9596-17 Smooth Infinite Economies by: J. Choi

by: M. Cragg

M. Kahn

by: C. Sanchirico

by: J. Bhagwati

A. Panagariya

by: W. Vickrey

by: C. Sanchirico

by: J. Choi

M. Thum

by: R. Findlay

by: J. Bhagwati

by: P. Dhrymes

by: P. Dhrymes

by: J. Choi

by: J. Mincer

by: P. Dhrymes

by: G. Chichilnisky

by: G. Chichilnisky

by: G. Chichilnisky 